\title{
Tumoración inguinal: una forma de presentación de la hidatidosis ósea. A propósito de un caso
} Inguinal tumor: a rare occurrence of hydatid disease. A case report

\author{
A.M. Calvo ${ }^{1}$, J.M. Cires ${ }^{1}$, S. Montón ${ }^{1}$, H. Sarasibar ${ }^{2}$, P. Lasanta ${ }^{3}$, F.J. Artázcoz ${ }^{3}$
}

\section{RESUMEN}

La enfermedad hidatídica ósea es una entidad infrecuente que representa el $0,5-2,5 \%$ de todas las hidatidosis. Las vértebras son los huesos más comúnmente afectados (50\%) seguidos de la pelvis (25\%) y de los huesos largos (15-25\%).

Las manifestaciones clínicas aparecen cuando existe una gran destrucción ósea o complicaciones, por lo que el diagnóstico se realiza generalmente en estadios tardíos mediante técnicas de imagen y en ocasiones de manera intraoperatoria.

El tratamiento en los casos operables es la extirpación quirúrgica pudiendo asociar tratamiento medico con albendazol.

Presentamos el caso de un paciente con hidatidosis ósea, localizada en la pelvis, en ausencia de afectación de otros órganos. El paciente presenta como única manifestación clínica una tumoración inguinal derecha y es intervenido quirúrgicamente ante la sospecha de una hernia inguinal complicada con el hallazgo intraoperatorio de hidatidosis pélvica.

Palabras clave. Tumoración inguinal. Hidatidosis ósea. Equinococcus granulosus.

\begin{abstract}
Osseous hydatid disease is an infrequent entity that represents $0.5-2.5 \%$ of all hydatidoses. The vertebrae are the most commonly affected bones (50\%), followed by the pelvis (25\%) and the long bones (15-25\%).

The clinical manifestations appear when there is large scale osseous destruction or complications, which is why the diagnosis is generally made in late stages through imaging techniques and on occasion in an intra-operational form.

Treatment in operable cases is by surgical extirpation, which can be associated with medical treatment with albendazol.

We present the case of a patient with osseous hydatidosis, localised in the pelvis, with an absence of other affected organs. The only clinical manifestation presented by the patient was an inguinal tumour on the right side. The patient received a clinical intervention facing the suspicion of an inguinal hernia, complicated by the intra-operational discovery of pelvic hydatidosis.
\end{abstract}

Key words. Inguinal tumour. Osseous hydatidosis. Equinococcus granulosus.

An. Sist. Sanit. Navar. 2007; 30 (3): 475-479.

1. Servicio de Cirugía General y Aparato Digestivo. Hospital García Orcoyen. Estella.

2. Servicio de Radiología.

3. Servicio de Cirugía Ortopédica y Traumatología. Hospital García Orcoyen. Estella.

Fecha de recepción el 19 de enero de 2007

Aceptado el 2 de marzo de 2007

Aceptación definitiva el 22 de junio de 200

\section{Correspondencia:}

Ana M. Calvo Benito

Servicio Cirugía General y Aparato Digestivo

Hospital de Navarra

C/ Irunlarrea, 3

31008 Pamplona

Tfno. 848422179 


\section{INTRODUCCIÓN}

La incidencia de hidatidosis en España es de 5 a 9 casos por cada 100.000 habitantes ${ }^{1,2}$. Es una infección parasitaria producida por la larva del Equinococcus granulosus que en su forma adulta vive en el intestino del perro y afecta al hombre al ingerir los alimentos contaminados por las heces de estos animales.

En el hombre tras la ingesta, los embriones de este parásito se liberan en el intestino delgado, atraviesan la mucosa intestinal y a través de la circulación portal son transportados (15\%) casi siempre al hígado (65\%) y al pulmón una vez sobrepasado el filtro hepático; si pasan el filtro de los capilares pulmonares y alcanzan la circulación arterial pueden afectar a otros órganos como el riñón, el cerebro o el sistema músculo esquelético $(0,5-2,5 \%)$ como se presenta en este caso ${ }^{3,4}$.

La hidatidosis ósea se caracteriza por un periodo de latencia largo (10-20 años), en el que no hay una alteración del estado general. De forma que se diagnostica en estadios muy avanzados, cuando hay una afectación muy extensa del hueso y su extirpación completa es muy difícil ${ }^{5}$.

Nuestro objetivo es describir una manifestación clínica poco usual de la enfermedad hidatídica.

\section{CASO CLÍNICO}

Varón de 76 años, agricultor, con antecedentes de hipertensión arterial, enfermedad pulmonar obstructiva crónica, fibrilación auricular y accidente cerebrovascular hace dos años, que consulta en el Servicio de Urgencias por presentar desde hace 3 días una tumoración inguinal derecha dolorosa. No refiere otras manifestaciones clínicas acompañantes.

En la exploración física el paciente presenta buen estado general con adecuada coloración e hidratación. La exploración de los aparatos respiratorio y cardiocirculatorio son normales y en el abdomen se aprecia una tumoración en la región inguinal derecha, dura, dolorosa e irreductible a la palpación.

Los datos de laboratorio muestran 10.600 leucocitos sin desviación izquierda y un $6 \%$ de eosinófilos. La radiografía simple de tórax y de abdomen (que no incluye toda la pelvis) no muestran anomalías.
Con la presunción diagnóstica de hernia inguinal derecha complicada, se interviene al paciente hallando durante el acto quirúrgico una tumoración quística de 4 por $4 \mathrm{~cm}$ de diámetro que infiltra la rama superior derecha del pubis y que al romperse de manera accidental contiene vesículas hidatídicas que se aspiran. Se realiza quistectomía parcial y lavado con suero hipertónico.

En el estudio anatomopatológico se evidencia la pared del quiste con fibrosis revestida en su parte interna por histiocitos y abundantes células gigantes multinucleadas y en su interior restos celulares, membranas hialinas acelulares y ganchos birrefringentes de escólex, lo que confirma la sospecha intraoperatoria de quiste hidatídico.

El postoperatorio transcurre sin incidencias y se realiza, durante el mismo, el estudio serológico con hemoaglutinación indirecta apreciándose títulos de $1 / 160$, una radiografía de pelvis en la que se halla una lesión lítica en la rama púbica superior derecha de aspecto multiloculado y expansiva que rompe la cortical (Fig. 1) y en la TC abdominal con contraste yodado endovenosos y oral se descarta la presencia de hidatidosis hepática y se objetiva la rama púbica superior derecha desestructurada con una lesión lítica que rompe la cortical anterior y un aumento de partes blandas adyacentes (Fig. 2).

En el momento del alta el paciente esta asintomático y se inicia tratamiento oral con albendazol $800 \mathrm{mg} /$ día, administrando ciclos de 28 días de tratamiento seguidos de 14 días de descanso hasta completar 3 ciclos.

En los controles posteriores el paciente presenta una limitación dolorosa a la movilización de la cadera en sus ejes máximos que no le impide la realización de una vida autónoma y que controla con analgésicos. En la radiografía de pelvis se aprecian múltiples lesiones quísticas de carácter infiltrativo que no respetan la cortical ósea en las ramas ilio e isquio pubianas de la hemipelvis derecha y la afectación del cotilo con incongruencia pero con espacio articular conservado. Se opta por tratamiento conservador y seguimiento periódico en consulta.

A los diez meses del diagnóstico, el paciente presenta un deterioro progresivo de sus capacidades cognitivas y de su relación con el medio, y tras sufrir una caída, ingresa en el hospital con dolor e impotencia a la deambulación.

En la radiografía de pelvis se aprecia una fractura de ramas pélvicas con afectación articular en la hemipelvis derecha sobre la zona de afectación de la hidatidosis, sin desplazamiento de los fragmentos. 


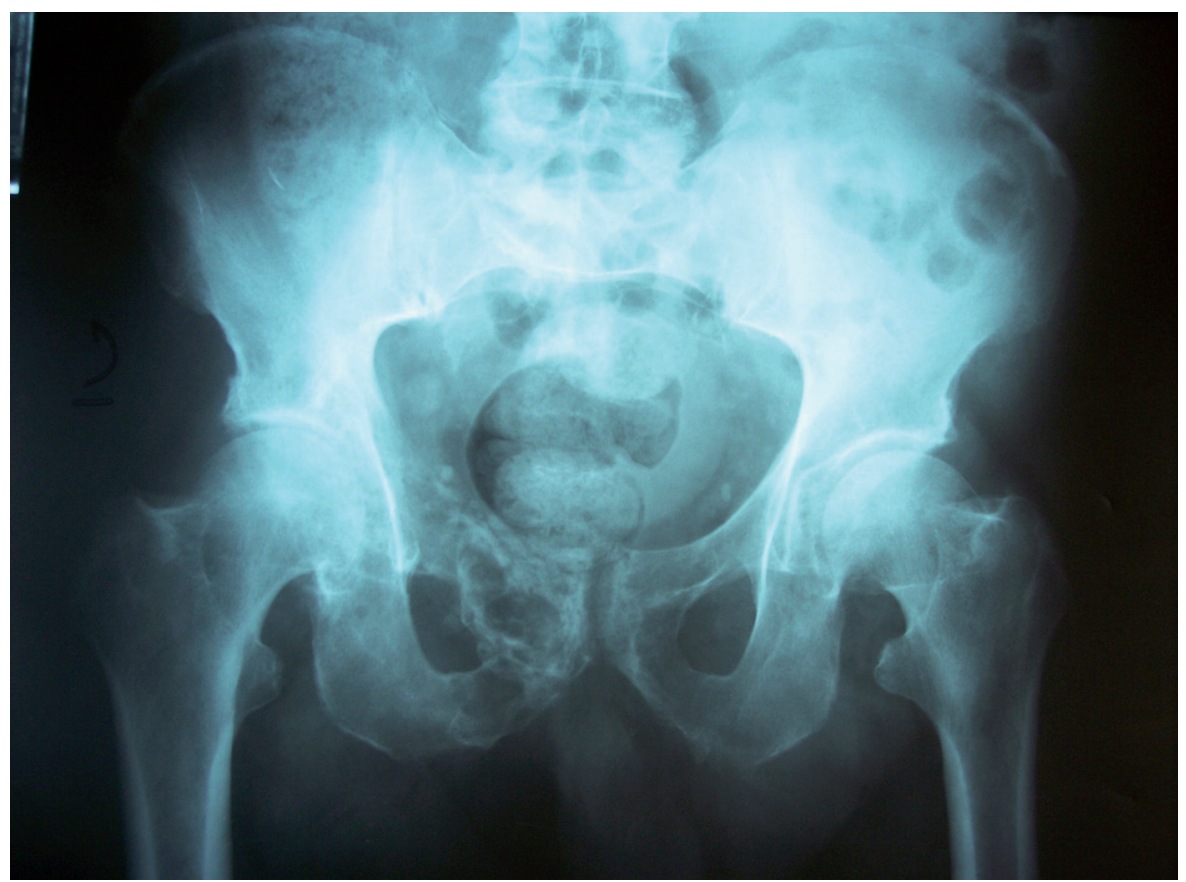

Figura 1. Rx de pelvis: lesión lítica en la rama púbica superior derecha.

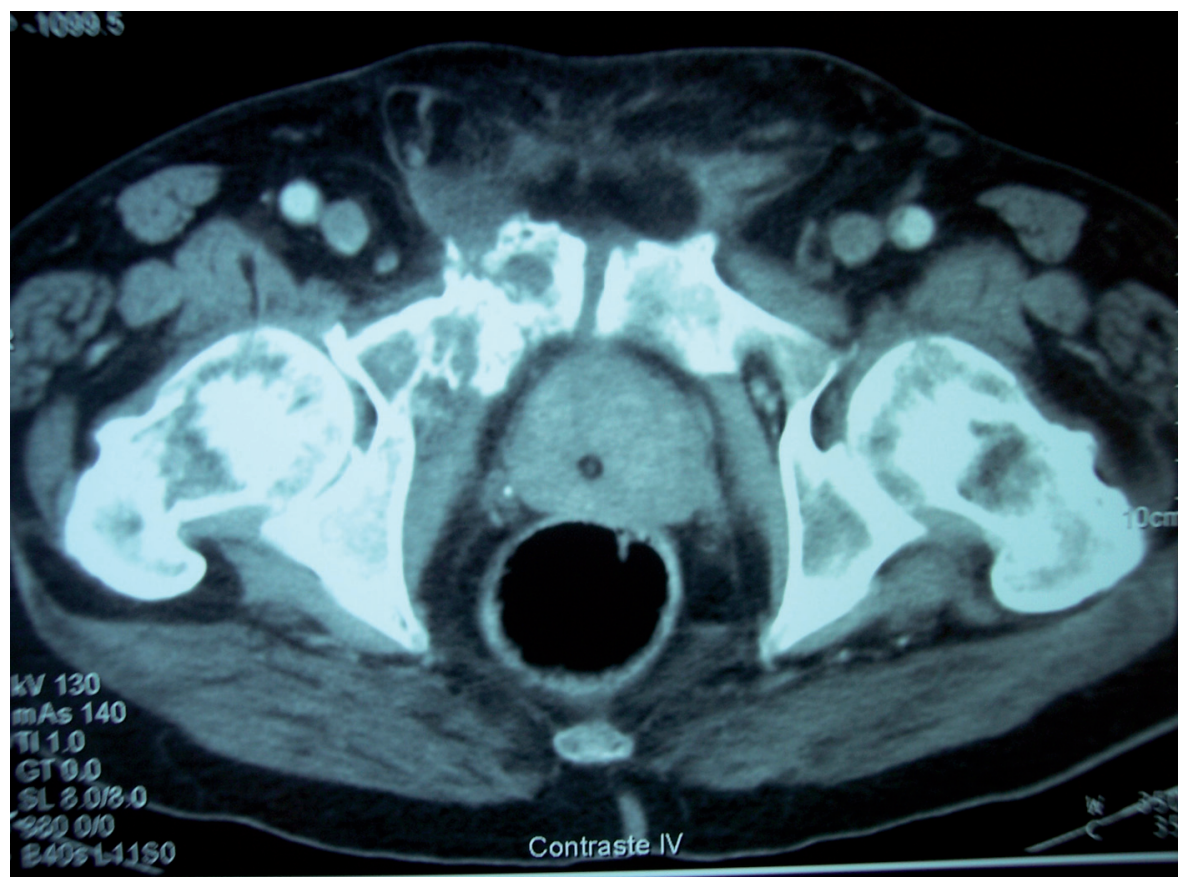

Figura 2. TAC abdominal: lesión lítica en la rama púbica superior derecha que rompe la cortical anterior con un aumento de partes blandas adyacentes. 
El realizar una sustitución articular por una prótesis total con una infección activa estaría contraindicada por el riesgo de diseminación de la parasitosis y la exéresis en bloque de la lesión e implante de una prótesis a medida con reconstrucción del acetábulo podría ser tenida en cuenta pero dado el deterioro cognitivo del paciente se opta por tratamiento conservador mediante deambulación de descarga y analgesia.

\section{DISCUSIÓN}

La hidatidosis es una enfermedad parasitaria de distribución universal debida a la larva del cestodo Equinococcus granulosus, cuyo huésped definitivo es el perro y que puede trasmitirse al hombre al ingerir alimentos contaminados por las heces de los perros parasitados ${ }^{6}$.

Las localizaciones primarias más frecuentes en el hombre son la hepática (65\%) y la pulmonar (15\%) siendo la hidatidosis ósea poco frecuente $(0,5-2,5 \%)^{7}$.

En la mayoría de los casos de hidatidosis ósea, ésta es la única localización de esta enfermedad y excepcionalmente se asocia a hidatidosis pulmonar o hepática, que deben ser descartadas durante el proceso diagnóstico con las técnicas de imagen habituales: ecografía hepática y radiografía de tórax ${ }^{8}$.

Las vértebras son los huesos más comúnmente afectados (50\%) seguidos de la pelvis (25\%) y de los huesos largos (15$25 \%)^{6,9}$.

La invasión intraósea ocurre por tres mecanismos: mecánico debido al crecimiento expansivo del quiste, fagocitario por los osteoclastos que se encuentran en las zonas de compresión e isquémico debido a la disminución de la circulación sanguínea ${ }^{9}$.

La hidatidosis ósea es una enfermedad silenciosa, de larga evolución ya que presenta un periodo de latencia largo (10-20 años); cuando aparece sintomatología clínica como dolor, tumefacción, inestabilidad o artralgias es debido a una destrucción ósea importante y si afecta a los tejidos adyacentes suelen aparecer tumoraciones de partes blandas ${ }^{8}$, como ocurre en nuestro caso. Si se localiza en la región inguinal es necesario el diagnóstico diferencial con otros procesos como la hernia inguinal complicada, lipomas, hematomas, adenopatías... Otras formas de presentarse esta enfermedad ósea es cuando aparecen complicaciones, como fracturas patológicas, infecciones bacterianas secundarias con fistulización, extensión transarticular e intrapelvica ${ }^{1,8}$.

Estos pacientes no suelen presentar alteraciones de los parámetros analíticos salvo eosinofilia en un $25 \%$ de los casos $^{8}$. Los estudios serológicos más sensibles son la hemoaglutinación indirecta o Elisa y los más específicos el Western blot o inmunoelectroforesis ${ }^{8,10}$ aunque la ausencia de serología positiva para Equinococcus no descarta la enfermedad.

Los estudios de imagen utilizados son las radiografías simples, la TC y la RMN. En la radiografía simple se observan lesiones líticas uni o multivesiculares de carácter expansivo, sin limites precisos que pueden romper la cortical y asociarse con un aumento de la densidad de las partes blandas adyacentes y calcificaciones al nivel de la periquística. Estos hallazgos son similares a los de la tuberculosis, abscesos, micosis y neoplasias ${ }^{3,11,12}$.

La TC y sobre todo la RMN, hoy en día considerada la prueba de elección, permiten valorar mejor las estructuras óseas afectadas y sus relaciones anatómicas ${ }^{5,6,13}$. Los hallazgos en la TC son similares a los de la radiografía simple y es un hallazgo típico en la RMN la lesión quística tabicada, hipointensa en T1 e hiperintensa en T2, pudiendo variar el grado de intensidad y la homogeneicidad según el contenido proteico y las vesículas hijas ${ }^{11,12}$.

El diagnóstico de certeza es encontrar escólex en la lesión resecada quirúrgicamente, como es nuestro caso $^{1,14}$ y la punción-biopsia no debe realizarse por el riego de rotura, extensión y anafilaxia que se presenta hasta en un $20 \%$ de casos $^{3}$.

El tratamiento de elección en los casos operables es la escisión completa con un margen amplio ya que si no va seguida de un elevado número de recidivas ${ }^{5,15} \mathrm{y}$ el tratamiento con mebendazol o albendazol puede asociarse a la cirugía o usarse como terapia aislada en las lesiones inoperables. Se recomienda albendazol $(10 \mathrm{mg} / \mathrm{Kg} /$ día $)$ ya que parece ser más eficaz que el 
mebendazol (40 mg/Kg/día) debido a su mayor penetración en los quistes ${ }^{16,17}$.

El pronóstico de la hidatidosis ósea es malo especialmente cuando afecta a las vértebras y la pelvis ya que la resección radical del área afectada es imposible en la mayoría de los pacientes ${ }^{3,7,18}$.

\section{BIBLIOGRAFÍA}

1. Zubiaurre L, OyarZabal I, Beguiristain A. Shock anafiláctico en pacientes con quiste hidatídico. Rev Esp Enferm Dig 2005; 97: 846-848.

2. Conthe P, Arnalich F, Muñóz JA, Segura JM, GIL A, BARBADO FS. La hidatidosis en un hospital general II. Estudio de 230 casos de hidatidosis hepática. Med Clin 1983; 80: 879-883.

3. BARREdo I, Aperribay M, CANCIO J. Hidatidosis ósea del peroné. Rev Esp Patol 2004; 37: 315320 .

4. Meyers WM, Neafie RC, Marty AM, Wear DJ, editors. Hydatidosis (Echinococcosis). En: Pathology of infectious diseases. Volume I. Helminthiases. Washinton DC: Armed Forces Institute of Pathology 2000: 145-164.

5. Torcal J, García-Álvarez G, Salinas JC, Sainz JM, Navarro A, GüEmEs A et al. Hidatidosis muscular primaria. Cir Esp 2002; 72: 147-151.

6. Roche F, Crusells MJ, Cuesta J, Letona S, SANJOAQUiN I, DE Miguel R et al. Hidatidosis ósea: a propósito de dos casos. Enferm Infecc Microbiol Clín 2002; 20: 134-135.

7. Massé A, Parola P, Brach del Prever EM, GalLINARO P. Hidatidosis of the pelvis: a case report with a 25-year follow-up. Arch Orthop Trauma Surg 2004; 124: 203-205.

8. Maguiña C, Guerra O, Ruiz C, Salinas C. Hidatidosis osteoarticular. Reporte de un caso. Rev Med Hered 2002; 13: 109-113.
9. Amoretti M, CARPIO J. Hidatidosis vertebral con extensión muscular y compresión medular. Reporte de un caso. Rev Peru Radiol 2003; 5: 38-40.

10. Zarzosa MP, Orduna Domingo A, Gutiérrez P, Alonso P, Cuervo M, Prado A et al. Evaluation of six serological test in the diagnosis and postoperative control of pulmonary hydatid disease patients. Diagn Microbiol Infect Dis 1999; 35: 255-262.

11. BegGS I. The radiology of hydatid disease. AJR Am J Roentgenol 1985; 145: 639-648.

12. Torricelli P, Martinelli C, Biagini R, Ruggieri P, DE CRISTOFAro R et al. Radiographic and computed tomographic findings in hydatid disease of bone. Skeletal Radiol 1990; 19: 435439.

13. Pedrosa I, Saiz A, Arrazika J, Ferreriros J, PEDROSA CS. Hydatid disease: radiologic and pathologic features and complications. Radiographics 2000; 20: 795-817.

14. Loudiye H, Aktaous S, Hassikou H, RL-Bardouni A, El-Manouar M, Fizazi M et al. Hydatid disease of bone. Review of 11 cases. Joint Bone Spine 2003. 70: 352-355.

15. Navarro AC, Torcal J, García-Álvarez F, BurDIO F, TEJERO E, GÜEMES A et al. Hidatidosis primaria recidivante del vasto interno. Cir Esp 2000; 68: 264-265.

16. MANEs E, SANTUCCI A. Echinococcosis: intramuscular localization. Chir Organi Mov 1990; 75: 189-196.

17. Prousalidis J, Tzardubigkiu K, Sgouradis L, KatSOHIS C, Aletras H. Uncommon sites of hydatid disease. Worlld J Surg 1998; 22: 1722.

18. Martínez AA, Herrera A, Cuenca J, Herrero L. Hidatidosis of the pelvis and hip. Int Orthop 2001; 25: 302-304. 
